\title{
Morphological Characteristics of a Rat Model of Reexpansion Pulmonary
} Edema

\author{
Shinichi Otani', Takashi Yashiro², Yasunori Sohara ${ }^{1}$ and Shunsuke Endo ${ }^{1 *}$
}

${ }^{1}$ Department of Thoracic Surgery, School of Medicine, Jichi Medical University, Yakushiji, Shimotsuke, Tochigi, Japan

${ }^{2}$ Division of Histology and Cell Biology, Department of Anatomy, School of Medicine, Jichi Medical University, 3311-1 Yakushiji, Shimotsuke, Tochigi, Japan

\section{Abstract}

Objective: Reexpansion pulmonary edema (RPE) is a severe disorder, and its pathophysiology is not well understood. One proposed mechanism for RPE is that chemical substances such as cytokines increase alveolar permeability. Another possible mechanism is that alveolar distention during reexpansion causes physical damage. To test the hypothesis that sudden alveolar distention damages alveolar cellular structure and identify the underlying cause of RPE, we developed and evaluated the morphological characteristics of a rat RPE model.

Methods: Lung from a rat model of RPE was observed by using live imaging from intravital fluorescence microscopy with fluorescein isothiocyanate labelled albumin as tracer, light microscopy, and electron microscopy (with and without horseradish peroxidase [HRP] as tracer).

Results: Intravital fluorescence microscopy and light microscopy showed that RPE developed almost immediately after lung reexpansion and that blood flow in pulmonary capillaries slowed substantially. In some capillaries, however, blood flow had stopped entirely; in others, anterograde and retrograde flow alternated. Electron microscopy revealed pores in type I pneumocytes and overt fissures in alveolar walls. Electron microscopic observation with HRP revealed that HRP moved from capillaries to the inner surfaces of alveolar epithelia in reexpanded lungs. In addition, diaminobenzidine reaction products from the HRP enzyme reaction were visible in areas with pores.

Conclusions: RPE occurred almost immediately after lung reexpansion. Pores developed in type I pneumocytes in alveolar epithelium. These pores, together with overt fissures in alveolar walls, allowed leakage of plasma components into alveoli. These findings appear to be important features of RPE development.

Keywords: Reexpansion pulmonary edema; Intravital fluorescence microscopy; Electron microscopy; Horseradish peroxidase; Rat model; Type I pneumocyte; Capillary

\section{Introduction}

Pulmonary edema is usually caused by hydrostatic imbalance or altered permeability of pulmonary blood vessels. Robin et al. [1] reported that edema caused by increased capillary pressure resulted from mitral valve insufficiency and other cardiac conditions that increase pressure inside the pulmonary vasculature, or from causes such as nephrotic syndrome, which can reduce plasma osmotic pressure. In contrast, edema caused by altered permeability results from an increase in the permeability of pulmonary microvasculature, which may be due to infection, acute respiratory distress syndrome, or the side effects of drugs, among other reasons. Reexpansion pulmonary edema (RPE) is caused by altered permeability.

RPE may develop during sudden reexpansion of a lung that has been collapsed for a prolonged period. Although RPE can result in respiratory failure, there is no entirely effective method of prophylaxis. Thus, it is important to improve our understanding of the pathophysiology of RPE. One putative mechanism involves chemical substances that might increase alveoli permeability, such as cytokines responsible for inflammation in pulmonary microvasculature [2]. Another potential mechanism is physical damage caused by sudden alveolar distention during reexpansion [3]. Unfortunately, the underlying mechanism for RPE is not well understood.

A characteristic of RPE is its sudden development after lung reexpansion [4]. This feature suggests that the more likely explanation for RPE is that sudden alveolar distention damages alveoli and/or alveolar cellular structure. To test this hypothesis and help identify the underlying cause of RPE, we developed and evaluated the morphological characteristics of a rat RPE model.

\section{Materials and Methods}

\section{Development of rat models of RPE and controls}

Wistar rats were purchased from Clea Japan (Tokyo, Japan). The animals were given conventional food and water ad libitum and were kept under a 12/12-hr light-dark cycle. Room temperature was maintained at approximately $22^{\circ} \mathrm{C}$. All animal experiments were performed after receiving approval from the Institutional Animal Experiments Committee of Jichi Medical University and were conducted in accordance with the Institutional Regulations for Animal Experiments and Fundamental Guidelines for Proper Conduct of Animal Experiments and Related Activities in Academic Research Institutions, under the jurisdiction of the Japanese Ministry of Education, Culture, Sports, Science and Technology (MEXT).

The rats (weight range: 280-330 g) were anesthetized by intraperitoneal injection of sodium pentobarbital, which was followed by orotracheal intubation. After being placed on their right side, the rats were put on a respirator (Model 683; Harvard Apparatus, Natick, MA, USA) and underwent thoracotomy on their left side between the fourth and fifth ribs. To facilitate edema development, the left bronchi

*Corresponding author: Shunsuke Endo, Department of Thoracic Surgery, Schoo of Medicine, Jichi Medical University, 3311-1, Yakushiji, Shimotsuke, Tochigi, Japan, Tel: +81-285-58-7368; Fax: +81-285-44-6271; E-mail: tcvshun@jichi.ac.jp

Received January 25, 2017; Accepted February 27, 2017; Published February 28, 2017

Citation: Otani S, Yashiro T, Sohara Y, Endo S (2017) Morphological Characteristics of a Rat Model of Reexpansion Pulmonary Edema. J Pulm Respir Med 7: 396. doi: 10.4172/2161-105X.1000396

Copyright: $\odot 2017$ Otani S, et al. This is an open-access article distributed under the terms of the Creative Commons Attribution License, which permits unrestricted use, distribution, and reproduction in any medium, provided the original author and source are credited. 
of the experimental group were first closed with clips. After atelectasis was confirmed, the incisions were closed and the rats were allowed to reawaken. The controls received sham surgery consisting of a similar thoracotomy without closing of the left bronchi.

Three days after these preliminary procedures, the rats were again anaesthetized, as described above, and tracheotomy was performed. The rats were placed on their right side, put on a respirator, and again underwent thoracotomy, during which the thoracic wall from the fourth to eighth ribs was removed to expose the lung. In the experimental group, the clips were then removed and the left lung was allowed to reexpand, resulting in RPE development in these animals.

\section{Intravital fluorescence microscopy}

The resulting RPE was visualized by intravital fluorescence microscopy with a modified version of the method of Endo et al. [5]. Ten milligrams of fluorescein isothiocyanate labelled bovine serum albumin (FITC-BSA; Sigma-Aldrich, St. Louis, MO, USA) was dissolved in $0.4 \mathrm{ml}$ distilled water and administered intravenously to the rats. In the lungs $(n=5)$ of the experimental group, clip removal from the left bronchi resulted in immediate reexpansion of the left lungs and RPE. To observe this in vivo, a vacuum chamber was used to secure the lungs for microscopic observation. The left lungs were placed in the vacuum chamber with the diaphragm side facing the glass cover of the chamber, which was set to a pressure of $-15 \mathrm{~cm} \mathrm{H} \mathrm{H}_{2} \mathrm{O}$, to replicate the intrathoracic conditions of the lung. After confirming that they contained air, the control lungs $(n=5)$ were simply placed in the vacuum chamber. The microcirculatory dynamics of left lungs were observed in vivo for 2 hours with an epifluorescence microscope (Optiphot2; Nikon, Tokyo, Japan) using 435-nm blue excitation light. During these observations, a CCD camera (C7190; Hamamatsu Photonics, Shizuoka, Japan) was used to obtain monochrome live images of the lungs.

\section{Light microscopy}

Approximately 1 minute after the left lungs of the experimental rats had reexpanded, they were excised and fixed in a $10 \%$ formaldehyde solution (0.1 M phosphate buffer, $\mathrm{pH}$ 7.4). Hematoxylin-eosin (HE)stained specimens were then created for light microscopic observation. The left lungs of the controls were excised approximately 1 minute after the thorax had been opened for the second time, followed by the above fixing and staining procedure for observation.

\section{Electron microscopy}

In accordance with the method of Kato et al. [6], the left lungs of experimental rats $(n=3)$ and controls $(n=3)$ were examined by electron microscopy, as follows. First, $10 \mathrm{ml}$ of $2 \%$ glutaraldehyde solution (0.1 M cacodylate buffer containing $2 \%$ sucrose, $\mathrm{pH} 7.4$ ) at $4^{\circ} \mathrm{C}$ was fed into the lungs through tracheotomy tubes at a pressure of $25 \mathrm{~cm}$ $\mathrm{H}_{2} \mathrm{O}$, after which left lungs were excised, and $5 \mathrm{~mm}$ thick slices were cut. Immersion fixation of these slices was then done for 120 minutes. These fixed lung tissue slices were then washed and thin-sliced into sections measuring about $3 \mathrm{~mm}^{2}$. These new sections were postfixed for 90 minutes by immersing them in $1 \% \mathrm{OsO}_{4}$ solution $(0.1 \mathrm{M}$ cacodylate buffer) at $4^{\circ} \mathrm{C}$. The sections were dehydrated with a graded series of ethanol and propylene oxide, after which the tissues were embedded in Epon resin. Ultrathin sections were prepared with an ultramicrotome and then stained with uranyl acetate and lead citrate. The sections were observed by using a transmission electron microscope (H7600; Hitachi, Tokyo, Japan).

\section{Electron microscopic observation of horseradish peroxidase tracer}

Immediately after removing the clips on the bronchi of three experimental rats, the movement of plasma components was observed with horseradish peroxidase (HRP) tracer (0.05 g; Type VI, SigmaAldrich) dissolved in $0.5 \mathrm{ml}$ of saline and intravenously administered over a period of 2 minutes. HRP was administered to control rats $(n=3)$ in the same manner, immediately after their thorax was opened. Six minutes after completing HRP administration, glutaraldehyde was fed into the lungs, as described above, and the left lungs were excised. Sections (thickness, $5 \mathrm{~mm}$ ) were cut and fixed using a method modified from Mabuchi et al. [7], including fixation, diaminobenzidine (DAB) reaction, postfixation, embedding, ultrathin sectioning, and electron staining. To improve the precision of $\mathrm{DAB}$ reaction, electron staining was first done by immersing the sections in a uranyl acetate water solution for only 1 minute and then in a lead citrate solution for only 1 minute.

\section{Results}

\section{Intravital fluorescence microscopic observation}

Intravital fluorescence microscopic observation of the pulmonary microvasculature yielded monochrome live images. When blue excitation light is shined on a specimen, the FITC-BSA marker appears as white. In contrast, areas with no FITC appear as black.

No FITC was seen in any alveoli from control specimens. All specimens appeared black because of the absence of fluorescence. The alveoli appeared as a valvate collection of oval structures. FITC appeared as white in pulmonary microvasculature, which formed a network around each alveolus. Inside these white-stained capillaries were numerous red blood cells, which were not fluorescent and therefore appeared as black dots. Observation confirmed that these cells flowed normally, without obstruction. The characteristics of these alveoli and capillaries did not change during the 2 hour observation period. Figures 1a-1c show time-lapse live imaging obtained during intravital fluorescence microscopic observation of lungs from the control group.

At 10 seconds after lung reexpansion, red spots were seen over the entire surface of left lungs from the experimental group. These findings suggest that reexpansion edema had caused widespread internal bleeding into alveoli in areas where these red spots developed. We concluded that RPE had developed very quickly while air was rushing into and reexpanding collapsed alveoli.

Fluorescence microscopy confirmed the above findings, which were visible grossly. In live imaging, approximately $80 \%$ of alveoli were white, and FITC had flowed from the surrounding pulmonary microvasculature into most alveoli. Furthermore, these conditions were largely unchanged during the 2 hour observation period. Blood flow had slowed in some capillaries of reexpanded lungs, and these capillaries were often adjacent to others with normal blood flow. In some capillaries, however, blood flow had stopped entirely; in others, anterograde and retrograde flow alternated. Overall, blood flow was slower in the pulmonary microvasculature. Inspection of the lungs from the start of reexpansion until 2 hours later revealed that blood flow was slightly slower at the end of the 2 hour period. Figures $2 d-2 f$ show still images from intravital fluorescence microscopic observation of lungs from the experimental group. 

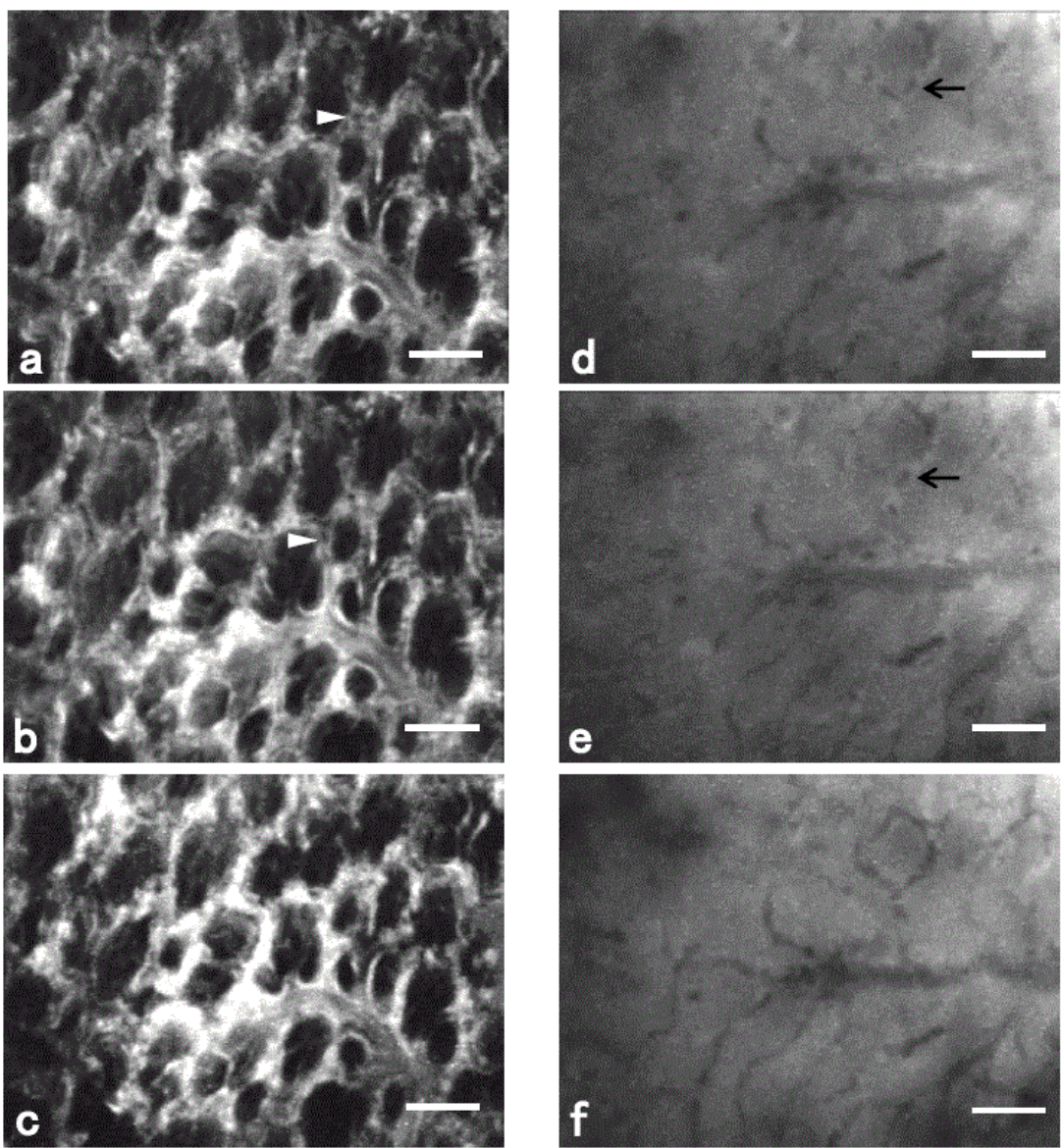

Figure 1: Time-lapse live images from in vivo fluorescence microscopic observation of lungs. a) Photograph of a lung from the control group. White areas within blood vessels indicate the presence of fluorescein isothiocyanate-labelled bovine serum albumin (FITC-BSA) marker. Red blood cells appear as black spots within the vessels. All alveoli are black, indicating the absence of FITC-BSA. b) A photograph obtained 0.23 seconds after (a). Arrowheads in (a) and (b) indicate identical red blood cells and show no evidence of flow disturbance. c) A photograph obtained 2 hours after (a). No changes over time are visible in alveoli or blood flow. d) Photograph of a lung from the experimental group. FITC-BSA can be seen leaking into alveoli. Blood vessels and alveoli appear white. Red blood cells appear as black dots inside blood vessels. e) A photograph obtained 0.23 seconds after (d). Arrows in (d) and (e) indicate identical red blood cells and show that flow is slower in these vessels than in control lung. f) A photograph obtained 2 hours after (d). No changes over time are visible with respect to leakage of FITC into alveoli. The number of red blood cells is increased in capillaries, and blood flow remains slow. Scale bar: $100 \mu \mathrm{m}$.

\section{Light microscopic observation}

The control group lungs exhibited the structure typical of normal lungs and had no visible abnormalities (Figures $2 \mathrm{a}$ and $2 \mathrm{~b}$ ). However, serum from surrounding microvasculature was present in most alveoli of experimental group lungs. Furthermore, red blood cells and alveolar macrophages were much more numerous in alveoli from experimental group lungs than in those from the control group (Figures $2 \mathrm{c}$ and $2 \mathrm{~d}$ ).

\section{Electron microscopic observation}

Electron microscopic observation of control lungs revealed normal lung structure and that sham surgery resulted in no abnormal changes (Figures $3 \mathrm{a}$ and $3 \mathrm{~b}$ ). In contrast, lungs from the experimental group all exhibited characteristic changes. First, almost no edematous changes were visible in alveolar walls. In addition, although the cytoplasm of most endothelial cells in capillaries near alveolar walls retained its normal, thin shape, the fine structure of the basement membrane forming the blood-air barrier was altered, as were type I pneumocytes in the alveolar epithelium. Figures $4 \mathrm{a}$ and $4 \mathrm{~b}$ show the basement membrane (which consists of a lamina densa layer sandwiched between two lamina lucida layers). The two lamina lucida layers had thickened slightly, especially the layer facing the blood vessels. Type I pneumocytes had numerous pores (diameter, 0.01-0.1 $\mu \mathrm{m}$ ) and had also thickened slightly. Furthermore, some of these pneumocytes appeared to be slightly damaged. As for microvascular endothelial cells in the area adjacent to where these pores had appeared in the alveolar epithelium, the cells in the microvasculature did not appear damaged, and their tight junctions were intact. Although such changes were not seen in all alveoli, they were very frequently observed. As for the blood-air barrier which consists of type I pneumocytes, the basement membrane, and vascular endothelial cells-large fissures were occasionally present at a position perpendicular to its three main layers. These fissures split the layered structure and allowed blood components to flow into alveoli (Figure 5).

\section{Electron microscopic observation of HRP tracer}

In the control group, $\mathrm{DAB}$ reaction products from the HRP enzyme reaction were present within the lumens of capillaries, but none were visible within alveolar lumens (Figures $6 \mathrm{a}$ and $6 \mathrm{~b}$ ). As shown in Figure $6 \mathrm{~b}$, numerous pinocytotic vesicles were visible in vascular endothelial cells. DAB reaction products were visible within these vesicles. There was also an interesting finding in the basement membrane of the blood-air barrier. This membrane comprises three layers-a lamina 

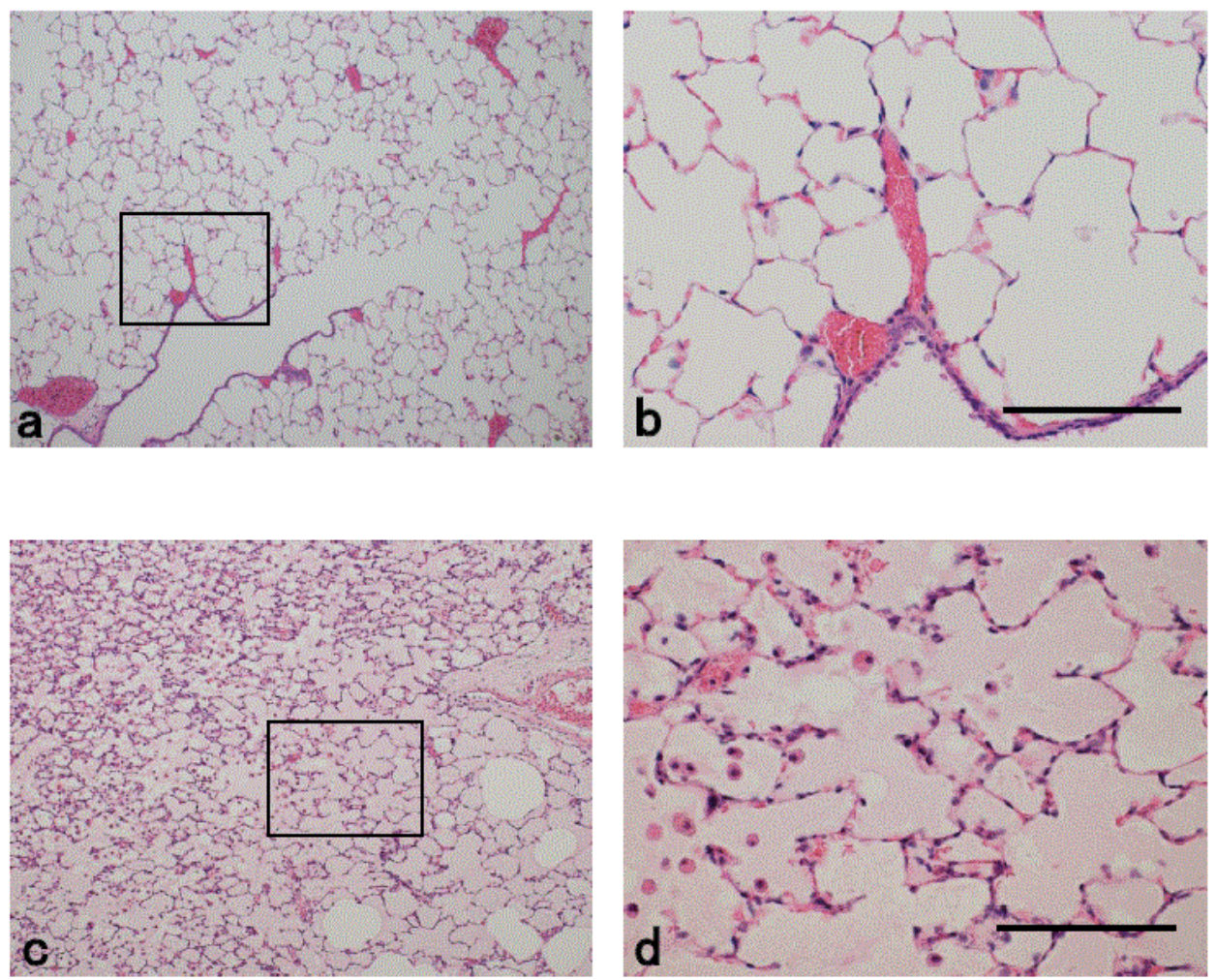

Figure 2: Light microscopic observation of HE-stained specimens of left lungs of rats. a) Images of the control group at low magnification. Terminal bronchioles, alveolar ducts, alveolar sacs, and alveoli are clearly visible. The lung structure appears normal, with no leakage of plasma components into alveoli. b) High-magnification view of the box in (a). Virtually no alveolar macrophages are present in alveoli, and no blood is leaking into alveoli. c) Lowmagnification view of reexpanded lung excised 1 minute after reexpansion. Plasma components are leaking into many alveoli. d) High-magnification view of the box in (c). A larger number of alveolar macrophages are visible in alveoli, as is blood leaking into alveoli. Scale bars: (b,d) $100 \mu \mathrm{m}$.

lucida layer on the vascular endothelial side, a lamina densa layer in the center, and another lamina lucida layer on the alveolar epithelium side. $\mathrm{DAB}$ reaction products were weakly visible in both lamina lucida layers and on the outer surfaces of these layers. In contrast, although there were pinocytotic vesicles in the thin cytoplasm of type I pneumocytes in the alveolar epithelium, no DAB reaction products were seen inside these vesicles.

In all experimental group lungs, DAB reaction products were visible over wide areas of reexpanded lungs and thinly covered the inner surfaces of alveolar epithelia (Figures $7 \mathrm{a}$ and $7 \mathrm{~b}$ ). In some alveoli, many pores were present in type I pneumocytes, and DAB reaction products were visible in areas with pores, down into the inner surfaces of alveoli. As described above, when HRP was not administered no remarkable changes were observed in vascular endothelial cells in adjacent capillaries, even where pores had formed in alveolar epithelium.

\section{Discussion}

We developed a rat model of RPE, which was first observed with fluorescence and light microscopy. Our findings revealed that RPE developed almost immediately after lung reexpansion and that blood flow in pulmonary capillaries slowed substantially. Electron microscopic observation (with and without HRP administration) revealed pores in type I pneumocytes and flow of blood plasma components into alveoli, both through these pores and through fissures in alveolar walls.

A previous in vivo study of RPE reported that blood flow slowed or stopped in pulmonary capillaries [8]. The present in vivo microscopic observations showed slower blood flow in capillaries of some areas of lungs with RPE, as well as areas in the same lungs in which flow was unchanged, others where it stopped, and others with recurrent retrograde blood flow (Figure $1 \mathrm{~b}$ ). Thus, although gross inspection suggested that edema was relatively evenly distributed over the entire surface of the lung, capillary hemodynamics varied substantially in areas affected by edema. The type of microscopic damage to alveolar walls varied. However, edema developed over virtually the entire lung surface, immediately after reexpansion (Figure 1b). Within 10 seconds of lung reexpansion, red spots were grossly visible on the surface. However, once edema had developed there was almost no change in the amount of serum seeping into alveoli at 2 hours later, which suggests that edema development was complete immediately after lung reexpansion.

A previous study reported that hypoxia-induced factor 1 (HIF-1) promoted expression of vascular endothelial growth factor (VEGF) [9] and that VEGF in turn promoted migration of monocytes and alveolar macrophages [10]. The present study showed that the number of alveolar macrophages increased inside alveoli immediately after RPE development (Figures $2 \mathrm{c}$ and $2 \mathrm{~d}$ ). This indicates that HIF-1 and VEGF were already present in tissue in the collapsed lung before reexpansion. Furthermore, as shown in Figure 2d, we observed bleeding in alveoli. The high volume of bleeding could not have occurred simply because of increased alveolar wall permeability. Instead, the bleeding likely happened in tandem with physical damage to alveolar walls, which was seen in some lung areas after reexpansion.

To our knowledge only one previous study used electron microscopy to examine the cause of RPE [3]. However, several studies 

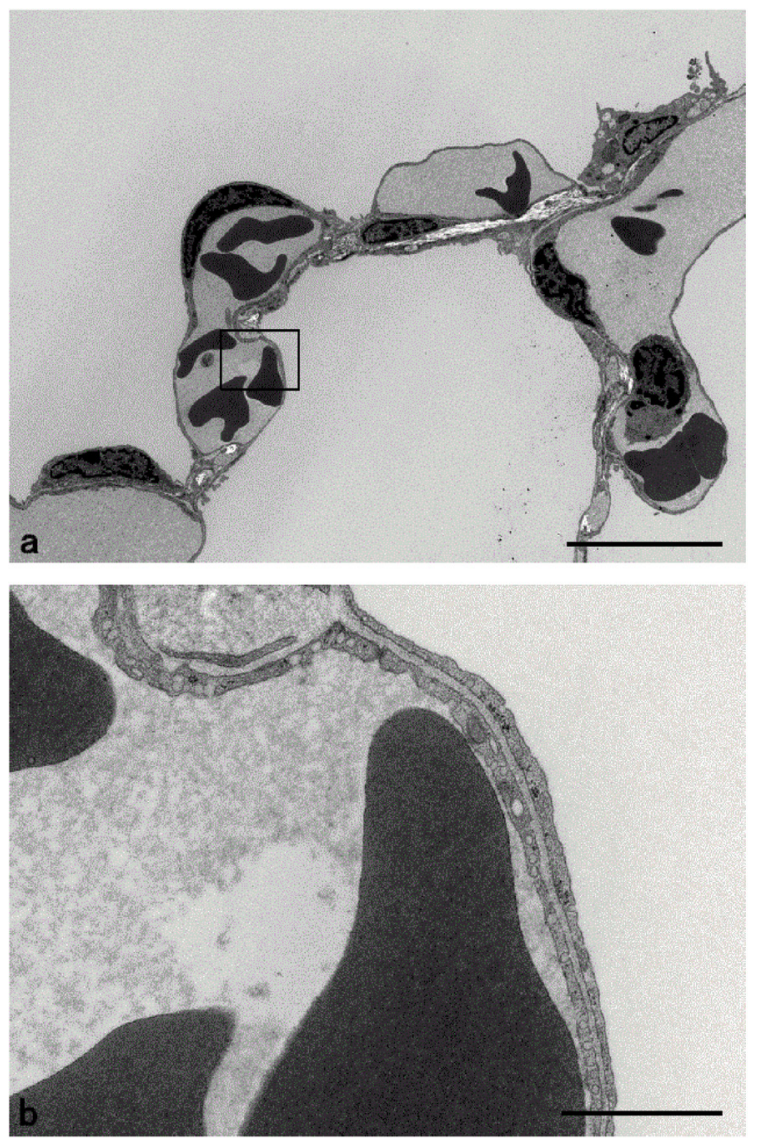

Figure 3: Electron microscopic images of rat lung from the control group. a) The image shows normal lung tissue and alveolar structure. b) High-magnification view of the box in (a). The structural components of the blood-air barrier are visible, including vascular endothelial cells, the basement membrane, and type I pneumocytes. Scale bars: (a) $10 \mu \mathrm{m}$, (b) $1 \mu \mathrm{m}$.

used electron microscopic observation to investigate pulmonary edema induced by causes other than lung reexpansion. Cottrell et al. [11] used electron microscopy to observe edema caused by static hydraulic lung pressure and reported that such edema only developed on alveolar walls in areas where collagen-fiber tissues were present (with no changes at the blood-air barrier). The same group analyzed electron microscopic images of alloxan-induced edema, which showed clasmatosis in the epithelium and endothelium but no edema in the alveolar septum [11]. The present experimental group exhibited almost no changes in vascular endothelial cells or in the distance between cells (Figures 3 and 4), but pores measuring $0.01-0.1 \mu \mathrm{m}$ in diameter were present in alveolar epithelial cells. No previous study reported similar changes in alveolar epithelial cells. In addition, the alveolar wall exhibited fissures perpendicular to its several layers, although the integrity of these layers was otherwise maintained (Figure 5). These changes in pulmonary tissue structure completely differ from those reported by Cottrell et al. [11] for other edema types (which were caused by alloxan or static hydraulic lung pressure). This discrepancy suggests that although pulmonary edemas might appear to have similar features, electron microscopic observation may yield very different findings, due to differences in pathogenesis.

One hypothesis regarding RPE pathogenesis maintains that chemical substances such as cytokines, and their action, change alveolar permeability in the pulmonary microvasculature. Another hypothesis suggests that physical damage from sudden alveolar distention during lung reexpansion results in edema. A study supporting the first hypothesis reported accumulation of white blood cells in alveoli, which resulted from decreased blood flow to and around the lung [12]. Another study reported damage to alveoli from increased concentrations of neutrophil elastase, which increases alveolar permeability [13], and suggested that reexpansion increased interleukin 8 and leukotriene B4 concentrations inside alveoli, thereby increasing vascular permeability [14]. One study reported that RPE in one lung is associated rarely with similar edema in the contralateral lung [15], which indicates that edema, at least in those cases, was not caused not by a physical mechanism (the contralateral lung did not undergo reexpansion) but was instead attributable to, for example, the chemical effects of cytokines.

The development of pores in pneumocytes (Figure 4) and fissures in alveolar walls (Figure 5) suggests that physical damage occurs during lung reexpansion and that such damage is the primary cause of RPE, at least initially. Other studies supporting a physical mechanism for edema, such as the study by Funakoshi et al., suggest that an increase in the number of inflammatory cytokines is not necessarily related to increased alveolar permeability [16]. Nakamura et al. reported that increased levels of polymorphonuclear leukocyte elastase reached a peak at around 19 hours after lung reexpansion and that this time point was not related to edema development [14]. The findings of the present study in which edema developed immediately after lung reexpansion suggest that cytokine action, or another chemical mechanism, is not the primary cause of RPE. Although the present results do not completely exclude the possible of effects of cytokines or other chemical factors as 

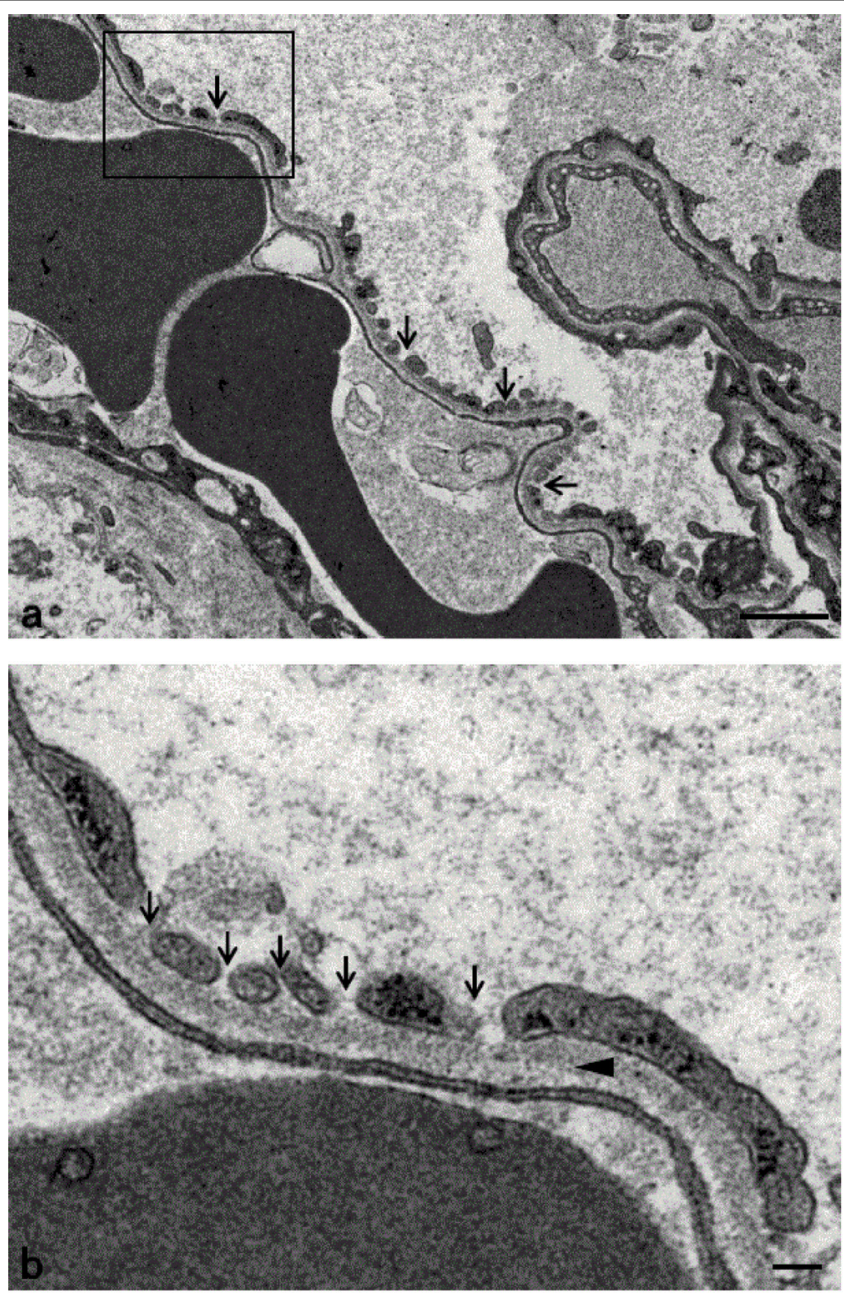

Figure 4: Electron microscopic images of rat lung from the experimental group. a) Numerous pores (arrows) are visible in some type I pneumocytes in reexpanded lung. b) High-magnification view of the box in (a) clearly showing 0.01 - to $0.1-\mu \mathrm{m}$ pores (arrows). Type I pneumocytes have become slightly thicker. There are no obvious changes in vascular endothelium. The arrowhead indicates the basement membrane, which is slightly thickened. Scale bars: (a) $1 \mu \mathrm{m}$, (b) $0.1 \mu \mathrm{m}$.

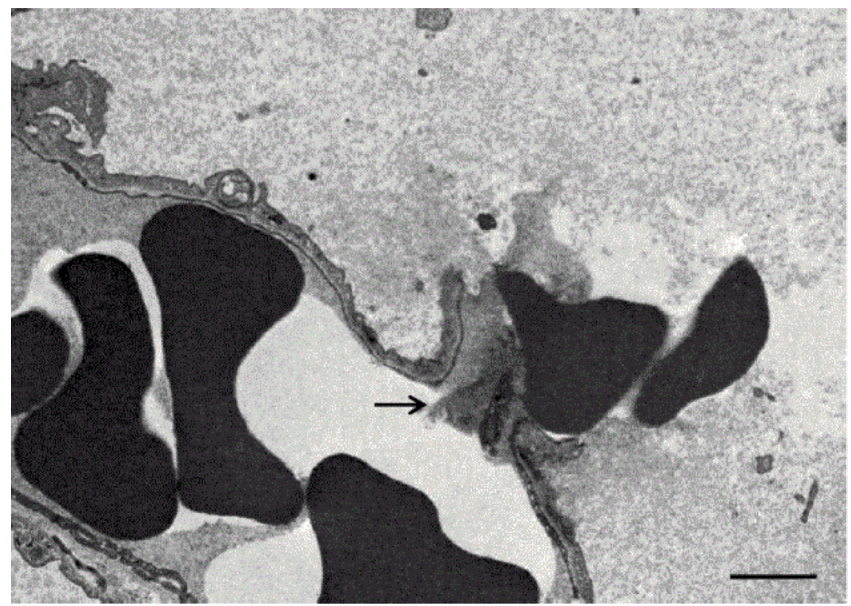

Figure 5: Electron microscopic images of rat lung from the experimental group. Perpendicular fissures are present in the alveolar wall and other layers of the blood-air barrier but do not otherwise disturb the layered structure; red blood cells leak into alveoli through these fissures (arrow). Scale bar: $1 \mu \mathrm{m}$.
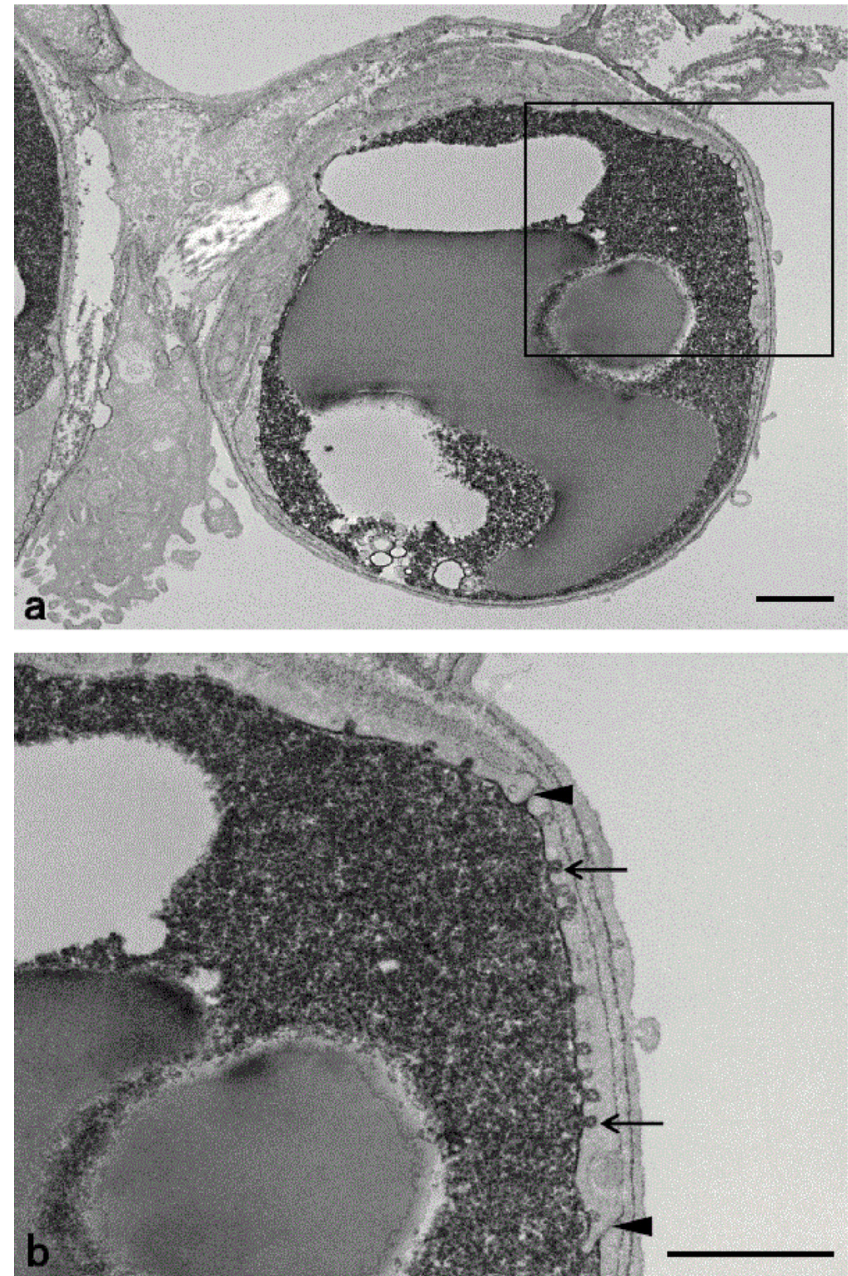

Figure 6: Electron microscopic images of rat lung from the control group, with HRP. a) The lumen of a capillary in a control lung is visibly filled with DAB reaction products, but none are present in alveoli. b) High-magnification view of the box in (a). Arrows indicate locations of pinocytotic vesicles with DAB reaction products. Arrowheads indicate the tight junction between endothelial cells. In some regions, DAB reaction products are visible in the putative lamina lucida of the basement membrane. Scale bars: $(a, b) 1 \mu \mathrm{m}$.

additional causes of RPE, the primary mechanism, at least for the initial manifestations, appears to be physical rather than chemical.

The addition of HRP tracer to our electron microscope observations allowed us to collect images of the actual movement of substances within pulmonary tissue. Simionescu et al. [17] reported that it takes 45 seconds for small molecules in pulmonary capillaries to move from these blood vessels through pinocytotic vesicles in vascular endothelial cells to the basement membrane [17]. Their findings are consistent with those in our control group (Figure 6). We also observed DAB reaction products in pinocytotic vesicles in the vascular endothelium and in the lamina lucida layer on the alveolar side of the basement membrane. However, no DAB reaction products were seen inside pinocytotic vesicles in alveolar epithelium or within alveolar lumens. This consistency in past and present findings suggests that our methods were suitable for the study of RPE.

Previous studies of fluid-overload pulmonary edema reported that blood did not leak from capillaries into alveoli, but rather from arterioles into bronchioles [18]. Moreover, in endotoxin-induced lung injuries blood leaks into alveoli from arterioles and venules, not from 
Citation: Otani S, Yashiro T, Sohara Y, Endo S (2017) Morphological Characteristics of a Rat Model of Reexpansion Pulmonary Edema. J Pulm Respir Med 7: 396. doi: 10.4172/2161-105X.1000396

Page 7 of 7
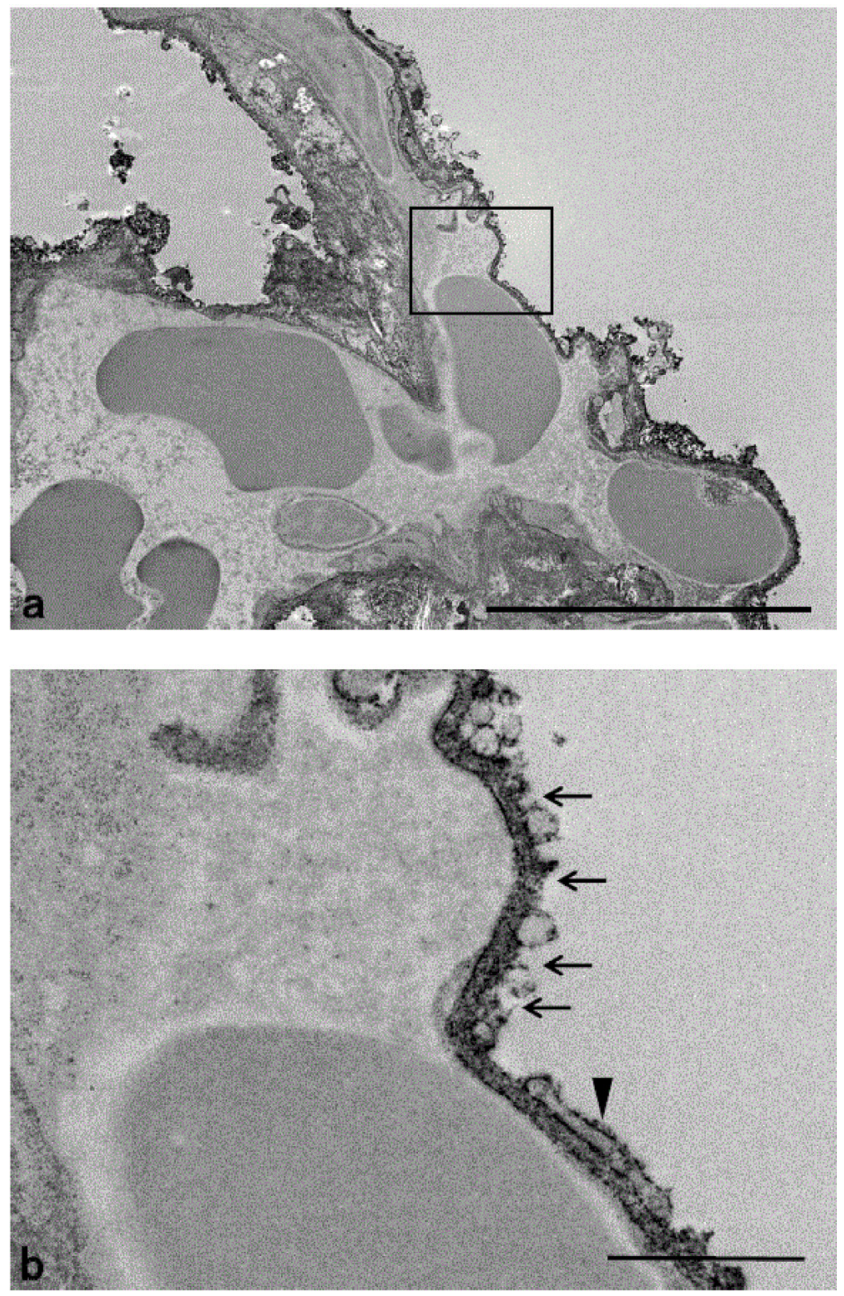

Figure 7: Electron microscopic images of a rat lung from the experimental group, with HRP. a) In this reexpanded lung, DAB reaction products are visible over a wide area of the interior surface of an alveolus. b) High-magnification view of the box in (a). Arrows indicate pores in pneumocytes of the alveolar epithelium. The arrowhead indicates $D A B$ reaction products on the inner surfaces of an alveolus. Scale bars: (a) $10 \mu \mathrm{m}$, (b) $1 \mu \mathrm{m}$.

capillaries [19]. The present study is the first to report that in RPE, HRP could pass from the basement membrane into alveoli (Figure 7), due to the presence of pores in alveolar epithelium. This finding substantially differs from the results of previous studies of other pulmonary conditions.

In summary, our findings show that RPE occurs almost immediately after lung reexpansion, that pores develop in type I pneumocytes in alveolar epithelium, and that these pores, together with overt fissures in the alveolar wall, allow leakage of plasma components into alveoli. These findings appear to be important features in RPE development.

\section{Acknowledgments}

We thank Tom Kouki (Department of Anatomy, Jichi Medical University School of Medicine) for his technical support, Morio Azuma (Department of Anatomy, Jich Medical University School of Medicine) for his editing support and David Kipler, ELS for revising the language of the manuscript.

\section{References}

1. Robin ED, Cross CE, Zelis R (1973) Pulmonary edema. 2. N Engl J Med 288: 292-304.
2. Meeker JW, Jaeger AL, Tillis WP (2016) An uncommon complication of common clinical scenario: exploring reexpansion pulmonary edema with a case report and literature review. J Community Hosp Intern Med Perspect 6: 32257

3. Sohara $Y$ (2008) Reexpansion pulmonary edema. Ann Thorac Cardiovasc Surg 14: 205-209.

4. Mahfood S, Hix WR, Aaron BL, Blaes P, Watson DC (1988) Reexpansion pulmonary edema. Ann Thorac Surg 45: 340-345.

5. Endo S, Sohara Y, Murayama F, Yamaguchi T, Hasegawa T, et al. (2003) Real-time visualization of partial liquid ventilation in a model of acute lung injury. Surgery 133: 207-215.

6. Kato T, Yashiro T, Murata Y, Herbert DC, Oshikawa K, et al. (2003) Evidence that exogenous substances can be phagocytized by alveolar epithelial cells and transported into blood capillaries. Cell Tissue Res 311: 47-51.

7. Mabuchi Y, Maruyama H, Yashiro T, Tanida S, Kurono C, et al. (1996) Sealing of the follicular lumen of the anterior pituitary gland of the male rat. Tissue Cell 28: $631-635$

8. Sohara Y, Endo S, Sato Y, Hasegawa T, Tetsuka K, et al. (2006) Vital observation of pulmonary microcirculation. J Jpn Soc Respir Endoscopy 28: 20-31.

9. Semenza GL1 (1998) Hypoxia-inducible factor 1: master regulator of O2 homeostasis. Curr Opin Genet Dev 8: 588-594.

10. Shen H, Clauss M, Ryan J, Schmidt AM, Tijburg P, et al. (1993) Characterization of vascular permeability factor/vascular endothelial growth factor receptors on mononuclear phagocytes. Blood 81: 2767-2773.

11. Cottrell TS, Levine OR, Senior RM, Wiener J, Spiro D, et al. (1967) Electron microscopic alterations at the alveolar level in pulmonary edema. Circ Res 21 783-797.

12. Thommasen HV, Martin BA, Wiggs BR, Quiroga M, Baile EM, et al. (1984) Effect of pulmonary blood flow on leukocyte uptake and release by dog lung. $J$ Appl Physiol Respir Environ Exerc Physiol 56: 966-974.

13. Suzuki S, Tanita T, Koike K, Fujimura S (1992) Evidence of acute inflammatory response in reexpansion pulmonary edema. Chest 101: 275-276.

14. Nakamura H, Ishizaka A, Sawafuji M, Urano T, Fujishima S, et al. (1994) Elevated levels of interleukin-8 and leukotriene B4 in pulmonary edema fluid of a patient with reexpansion pulmonary edema. Am J Respir Crit Care Med 149: $1037-1040$

15. Heller BJ, Grathwohl MK (2000) Contralateral reexpansion pulmonary edema. South Med J 93: 828-831.

16. Funakoshi T, Ishibe Y, Okazaki N, Miura K, Liu R, et al. (2004) Effect of re expansion after short-period lung collapse on pulmonary capillary permeability and pro-inflammatory cytokine gene expression in isolated rabbit lungs. $\mathrm{Br}$ Anaesth 92: 558-563.

17. Simionescu N, Simionescu M, Palade GE (1973) Permeability of muscle capillaries to exogenous myoglobin. J Cell Biol 57: 424-452.

18. Yoneda K (1980) Anatomic pathway of fluid leakage in fluid-overload pulmonary edema in mice. Am J Pathol 101: 7-16.

19. Nagata N, Sueishi K, Tanaka K (1991) Anatomic pathway of pulmonary fluid leakage in endotoxemia induced in rats. The red blood cell packing method and its application. Am J Pathol 138: 183-193. 